\title{
Expanding therapeutic frontiers in metastatic HER2-positive breast cancer
}

\author{
Sandra Cuellar \\ University of Illinois at Chicago, College of Pharmacy, Chicago, IL, USA \\ Correspondence to: Sandra Cuellar, PharmD, BCOP. University of Illinois at Chicago, College of Pharmacy, Chicago, IL, USA. Email: scuell1@uic.edu. \\ Comment on: Yan M, Bian L, Hu X, et al. Pyrotinib plus capecitabine for human epidermal growth factor receptor 2-positive metastatic breast cancer \\ after trastuzumab and taxanes (PHENIX): a randomized, double-blind, placebo-controlled phase 3 study. Transl Breast Cancer Res 2020;1:13.
}

Received: 28 July 2020; Accepted: 11 August 2020; Published: 31 January 2021.

doi: $10.21037 /$ tbcr-20-48

View this article at: http://dx.doi.org/10.21037/tbcr-20-48

Extraordinary therapeutic advances have been made in the management of HER2-positive metastatic breast cancer (MBC) over the past 2 decades. The approval of pertuzumab, ado-trastuzumab emtansine, lapatinib, neratinib, trastuzumab deruxtecan, and tucatinib have demonstrated the continued successful outcomes and importance of targeting HER2. Unfortunately, despite these therapeutic advances, nearly all patients with metastatic HER2-positive breast cancer eventually will progress on anti-HER2 therapy due to de novo or acquired resistance. Unraveling resistance mechanisms to anti-HER2 therapies as well as unveiling compensatory pathways and tumor heterogeneity is essential for the development of novel therapeutic strategies.

\section{Pyrotinib: mechanism of action \& phase I data}

Pyrotinib is an oral, irreversible pan-ERb receptor tyrosine kinase inhibitor with activity against epidermal growth factor receptor (EGFR)/HER1, HER2, and HER4. By covalently binding with ATP binding sites of intracellular regions, the drug inhibits the formation of homologous/ heterodimer and auto-phosphorylation of HER family, thus blocking the activation of RAS/RAF/MEK/MAPK, PI3K/AKT signaling pathways and tumor cell cycle in G1 phase and restricting tumor development (1). Data in the preclinical setting suggest that pyrotinib can irreversibly inhibit multiple ErbB receptors and effectively inhibit the proliferation of HER2-overexpressing cells both in vivo and in vitro. The phase I study determined the MTD of pyrotinib was $400 \mathrm{mg}$ daily. The study also suggested that pyrotinib is safe and effective in patients with HER2positive $\mathrm{MBC}$, with an overall response rate (ORR) of $50.0 \%$ and a median-progression free survival (PFS) of 35.4 weeks in the dosage range of 80 to $400 \mathrm{mg}$ daily. The phase I study also investigated biomarkers such as PIK3CA and TP53 mutations in circulating tumor DNA (ctDNA) led to worse efficacy with pyrotinib monotherapy (2).

\section{Pyrotinib: efficacy in phase II and phase III studies}

The efficacy of pyrotinib in combination with capecitabine in patients with HER2-positive MBC has been investigated in both phase II and phase III clinical trials. The efficacy in patients previously treated with taxanes, anthracyclines and/or trastuzumab was demonstrated in a randomized, open-label, active comparator-controlled, multicenter phase II trial. The primary endpoint was objective response rate which was significant greater with pyrotinib plus capecitabine compared to lapatinib plus capecitabine, as assessed by the investigator ( $79 \%$ vs. $57 \% ; \mathrm{P}=0.01)$ Independent imaging assessment reported similar findings ( $71 \%$ vs. 49\%; $\mathrm{P}=0.0117$ ). Other efficacy endpoints reported included duration of response and PFS. Duration of response was 16.7 months with pyrotinib plus capecitabine and 8.4 months with lapatinib plus capecitabine (hazard ratio 0.404). In terms of PFS, pyrotinib plus capecitabine significantly prolonged median PFS versus lapatinib plus capecitabine (18.1 vs. 7.0 months; adjusted HR 0.363; 95\% CI, 0.228-0.579; $\mathrm{P}<0.0001$ ) (3). At the 2020 American Society of Clinical Oncology annual meeting, interim 
results of the phase III PHOEBE study were presented. The PHOEBE trial enrolled patients who had already received treatment with trastuzumab and taxanes, with or without anthracyclines, and up to two lines of chemotherapy for metastatic disease (median, 1). Participants were randomly assigned to receive pyrotinib $400 \mathrm{mg} /$ day $(\mathrm{n}=134)$ or lapatinib $1,250 \mathrm{mg} / \mathrm{day}(\mathrm{n}=132)$, both given alongside capecitabine $1,000 \mathrm{mg} / \mathrm{m}^{2}$ twice a day on days $1-14$ of every 21-day cycle.

At a median follow-up of 9.9 months, the primary endpoint of PFS by independent review was significantly improved with pyrotinib versus lapatinib, at a median of 12.5 and 6.8 months, respectively, giving a hazard ratio of 0.39 in favor of pyrotinib.

Pyrotinib was favored across all subgroups, although the PFS benefit did not reach statistical significance for some subgroups, such as patients with trastuzumab resistance (defined as relapse within 6 months of adjuvant use and/or within 3 months in the metastatic setting) and those with non-visceral metastases.

The objective response rate was higher in the pyrotinib than lapatinib treatment arm, at $67.2 \%$ versus $51.5 \%$ (complete responses in $5.2 \%$ vs. $0.8 \%$ ), as was the clinical benefit rate, at $73.1 \%$ versus $59.1 \%$. The median durations of response were 11.1 and 7.0 months, respectively, and a corresponding $70.0 \%$ and $48.5 \%$ of responses were ongoing at data cutoff.

Overall survival (OS) data had not reached maturity (4). In August 2018, pyrotinib was approved in combination with capecitabine for the treatment of HER2-positive MBC in patients previously treated with anthracycline or taxane in China.

\section{PHENIX study}

In this issue of Translational Breast Cancer Research, Yan and colleagues evaluated the use of pyrotinib (400 $\mathrm{mg}$ orally once daily) in combination with capecitabine $\left(1,000 \mathrm{mg} / \mathrm{m}^{2}\right.$ BID on days 1-14) for 21 day cycle compared to placebo/ capecitabine in MBC patients with pretreated trastuzumab/ taxane. Patients who progressed on placebo plus capecitabine received subsequent pyrotinib monotherapy. The primary endpoint was PFS per independent review; 185 patients were randomly assigned to the pyrotinib arm versus 94 in the placebo arm. The median PFS was 11.1 months (95\% CI, 9.7-16.5) vs. 4.1 months (95\% CI, 2.8-4.2) in the pyrotinib $v s$. placebo groups with a reported hazard ration, 0.18 (95\% CI, 0.13-0.26); $\mathrm{P}<0.001$. Seventy-one patients in the placebo group subsequently received pyrotinib, showing a response rate of $38 \%$ (95\% CI, 26.7-49.3\%) and median PFS of 5.5 months (95\% CI, 4.1-6.9). In terms of toxicity, the most frequent grade 3 or 4 treatment related adverse events were diarrhea and hand-foot syndrome (5).

\section{PHENIX study applications in real world}

This study demonstrates the efficacy of a novel oral treatment regimen for the management of HER2 positive breast cancer after failure of trastuzumab/taxane. Application of the data in a global perspective would be limited, as the study population was not previously treated with combination pertuzumab/trastuzumab plus taxane in the $1^{\text {st }}$ line setting or treated in the second line setting with ado-trastuzumab or more recently US approved combination consisting of tucatinib/capecitabine/ trastuzumab. These regimens are considered preferred and standard of care in the US. However, because of cost and availability of pertuzumab, ado-trastuzumab, and other oral HER2 directed tyrosine kinase inhibitors (tucatinib, neratinib, lapatinib) these agents are not options for all patients in China. In addition to drug access issues, the COVID-19 pandemic has increased interest in toward a complete oral regimen. Therefore, other treatment options are needed after failure of trastuzumab/taxane or in the clinical situation where trastuzumab would be contraindicated because of cardiac issues and limited access to other trastuzumab directed therapies. The study design in the PHENIX supports treatment paradigms in China and other real world case scenarios where there is limited use of pertuzumab and ado-trastuzumab. In addition, data in China suggest that utilization of trastuzumab is also limited because of cost. This is an important detail, as optimal comparator arm would have been the combination of capecitabine plus trastuzumab or other HER2 directed therapy compared to capecitabine plus pyrotinib. The PHENIX study reported impressive improved PFS among all subsets of patients, including those with CNS metastases and visceral metastasis. In addition, pyrotinib demonstrated monotherapy activity after progression of capecitabine, with an impressive PFS and ORR. This monotherapy activity, although seen in a small subset of patients, suggests the potency of pyrotinib and benefit in a monotherapy setting. This is novel, given other oral HER2 directed agents have demonstrated limited efficacy as monotherapy. In terms of toxicity, diarrhea management is critical. Pyrotinib increases diarrhea in combination with capecitabine and was also 
observed as monotherapy (all grades $88.7 \%$, grade 3 and 4 $22.5 \%)$.

\section{Conclusions}

The PHENIX study provides efficacious and tolerable oral regimen in a real world setting in which costly HER2 directed agents are not available, limited access, contraindicated, or preference of an oral regimen secondary to COVID-19. The combination demonstrated efficacy across all subset of patients, including those with CNS involvement. Currently there are three to four options combining HER2 directed tyrosine kinase inhibitor with capecitabine, depending on drug approvals. Headto-head data in the PHOEBE trial suggest pyrotinib/ capecitabine has improved PFS compared to the lapatinib arm. However, we are lacking head-to-head data with neratinib and tucatinib. Therefore, it would be difficult at this time to have a preferred HER2 TKI combined with capecitabine. In addition, to efficacy and safety data, other considerations in selection of an anticancer regimen would be cost. Economic evaluation of these novel capecitabine combinations should also be a consideration in selection of treatment options for patients. In summary, the PHENIX data contributes to the other emerging data of the potent activity seen with pyrotinib in HER2 positive MBC and offers additional treatment options to patients diagnosed with HER2-positive breast cancer.

\section{Acknowledgments}

Funding: None.

\section{Footnote}

Provenance and Peer Review: This article was commissioned by the Editorial Office, Translational Breast Cancer Research. The article did not undergo external peer review.

Conflicts of Interest: The author has completed the ICMJE uniform disclosure form (available at http://dx.doi. org/10.21037/tbcr-20-48). The author has no conflicts of interest to declare.

Ethical Statement: The author is accountable for all aspects of the work in ensuring that questions related to the accuracy or integrity of any part of the work are appropriately investigated and resolved.

Open Access Statement: This is an Open Access article distributed in accordance with the Creative Commons Attribution-NonCommercial-NoDerivs 4.0 International License (CC BY-NC-ND 4.0), which permits the noncommercial replication and distribution of the article with the strict proviso that no changes or edits are made and the original work is properly cited (including links to both the formal publication through the relevant DOI and the license). See: https://creativecommons.org/licenses/by-nc-nd/4.0/.

\section{References}

1. Xuhong JC, Qi XW, Zhang Y, et al. Mechanism, safety and efficacy of three tyrosine kinase inhibitors lapatinib, neratinib and pyrotinib in HER2-positive breast cancer. Am J Cancer Res 2019;9:2103-19.

2. Ma F, Li Q, Chen S, et al. Phase I Study and Biomarker Analysis of Pyrotinib, a Novel Irreversible Pan-ErbB Receptor Tyrosine Kinase Inhibitor, in Patients With Human Epidermal Growth Factor Receptor 2-Positive Metastatic Breast Cancer. J Clin Oncol 2017;35:3105-12.

3. Ma F, Ouyang Q, Li W, et al. Pyrotinib or Lapatinib Combined With Capecitabine in HER2-Positive Metastatic Breast Cancer With Prior Taxanes, Anthracyclines, and/or Trastuzumab: A Randomized, Phase II Study. J Clin Oncol 2019;37:2610-9.

4. Xu B, Yan M, Ma F, et al. Pyrotinib or lapatinib plus capecitabine for HER2 + metastatic breast cancer (PHOEBE): A randomized phase III trial. J Clin Oncol 2020;38:1003.

5. Yan M, Bian L, Hu X, et al. Pyrotinib plus capecitabine for human epidermal growth factor receptor 2-positive metastatic breast cancer after trastuzumab and taxanes (PHENIX): a randomized, double-blind, placebocontrolled phase 3 study. Transl Breast Cancer Res 2020;1:13.

doi: $10.21037 /$ tbcr-20-48

Cite this article as: Cuellar S. Expanding therapeutic frontiers in metastatic HER2-positive breast cancer. Transl Breast Cancer Res 2021;2:1. 